\title{
A free-boundary problem for Stokes equations: classical solutions
}

\author{
S. ANTONTSEV \\ Universidade da Beira Interior, 6200 Covilhã, Portugal and Lavrentiev Institute of \\ Hydrodynamics, 630090 Novosibirsk, Russia \\ A. Meirmanov \\ Universidade da Beira Interior, 6200 Covilhã, Portugal \\ AND \\ B. V. YURINSKY \\ Universidade da Beira Interior, 6200 Covilhã, Portugal and Sobolev Institute of Mathematics, \\ 630090 Novosibirsk, Russia \\ [Received 18 June 1999 and in revised form 9 December 1999]

\begin{abstract}
The problem considered is that of evolution of the free boundary separating two immiscible viscous fluids with different constant densities. The motion is described by the Stokes equations driven by the gravity force. For flows in a bounded domain $\Omega \subset \mathbb{R}^{n}, n \geqslant 2$, we prove existence and uniqueness of classical solutions, and concentrate on the study of properties of the moving boundary separating the two fluids.
\end{abstract}

\section{The main result}

\subsection{Posing the problem}

We consider a flow of two immiscible viscous fluids with different constant densities in a bounded domain $\Omega \subset \mathbb{R}^{n}$ with a $C^{2}$-smooth boundary $S=\partial \Omega$ for dimensions $n \geqslant 2$. The evolution is driven by the gravity force. The moving boundary, which appears naturally, separates the subdomains occupied by different fluids.

More precisely, the problem is to find velocity $u=\left(u_{1}, \ldots, u_{n}\right) \in \mathbb{R}^{n}$, pressure $p \in \mathbb{R}$ and density $\rho \in \mathbb{R}$ from the system of equations for velocity and pressure:

$$
\begin{aligned}
\Delta u & =\nabla p+\alpha \rho \mathbf{e}, \\
\operatorname{div} u & =0,
\end{aligned}
$$

where $\mathbf{e}$ is a given unit vector and $\alpha=$ const a given scalar constant, and the transport equation for density is

$$
\frac{\mathrm{d} \rho}{\mathrm{d} t} \equiv \frac{\partial \rho}{\partial t}+\operatorname{div}(\rho u)=\frac{\partial \rho}{\partial t}+u \cdot \nabla \rho=0 .
$$

Time $t$ enters the equation for velocity as a parameter, so this needs no initial condition. The boundary condition on the known part of the boundary is

$$
\left.u\right|_{S}=0 .
$$


At the initial moment $t=0$, the density is piecewise constant and assumes two positive values characterizing the distinct phases of the flow

$$
\rho(x, 0)=\rho_{0}(x)=\left\{\begin{array}{ll}
\rho^{+}, & x \in \Omega^{+}(0), \\
\rho^{-}, & x \in \Omega^{-}(0),
\end{array} \quad \rho^{ \pm}=\text {const }, \quad \rho^{+}>\rho^{-}>0 .\right.
$$

In this case, the initial condition for density is equivalent to specifying the surface $\Gamma(0)=\Gamma_{0}$ that separates the two subdomains $\Omega^{ \pm}(0)$ initially occupied by different fluids. This surface is supposed to be sufficiently regular in the sequel.

The problem treated here is that of finding $u, p$ and the density $\rho(x, t)$ from the above equations and initial data. Note that it is non-linear because of the coupling term $u \cdot \nabla \rho$ in (1.3).

It is shown below that the evolution described by the above equations preserves the existence of two subdomains $\Omega^{ \pm}(t)$, each occupied by one of the fluids, that are separated at time $t>0$ by a regular free boundary $\Gamma(t)$. Thus, the problem studied is equivalent to finding $u, p$ and the moving boundary $\Gamma(t)$.

Theorems on the existence of generalized solutions to the Navier-Stokes system for nonhomogeneous incompressible fluids were obtained in, e.g., [1,2, 5, 6, 9, 13,15] (without detailed analysis of the set where the density is discontinuous).

\subsection{The main result}

Our principal result is the following theorem.

THEOREM 1.1 If the initial configuration of the free boundary is a surface $\Gamma_{0}$ of class $C^{2}$, then the problem (1.1)-(1.5) with piecewise constant initial density has a unique solution on the interval $[0, T]$ of arbitrary length $T>0$. The elements of this solution enjoy the following properties.

(i) For an arbitrary $q>n$ and $\lambda=1-\frac{n}{q}$, the velocity satisfies the relation

$$
u \in L^{\infty}\left(0, T ; W^{2, q}(\Omega)\right) \cap L^{\infty}\left(0, T ; C^{1, \lambda}\right) \cap C^{0, \lambda}\left(0, T ; C^{1, \lambda}\right) .
$$

(ii) The free boundary $\Gamma(t)$ is a surface of class $C^{1, \lambda}$ at each time $t \in(0, T]$, and the velocity $V_{\mathbf{n}}(x, t)$ of the free boundary in the direction of its normal $\mathbf{n}$ at position $x$ is uniformly bounded:

$$
\sup _{\substack{t \in(0, T) \\ x \in \Gamma(t)}}\left|V_{\mathbf{n}}(x, t)\right|<\infty
$$

(iii) The density has bounded variation

$$
\rho \in L^{\infty}(0, T ; B V(\Omega)) \cap B V\left(\Omega_{T}\right), \quad \Omega_{T}=\Omega \times(0, T) .
$$

Throughout the article, we use the customary notation of function spaces and norms (see, e.g., [4]). Thus, $W^{2, q}(\Omega)$ is the Sobolev space of functions with derivatives summable to power $q$ and $C^{k, \lambda}$ consists of functions whose $k$ th derivatives satisfy the Hölder condition with exponent $\lambda$. As usual, $a \cdot b$ is the scalar product of $a$ and $b, \nabla_{i} \varphi \equiv \frac{\partial}{\partial x_{i}} \varphi, \nabla \varphi=\left(\nabla_{j} \varphi\right) \in \mathbb{R}^{n}$ is the gradient, etc. 


\section{Proof of the main result}

We divide the proof of Theorem 1.1 into several steps.

First, we show that the problem of finding $u$ and $\rho$ has at least one classical solution if the initial density is smooth, $\rho_{0} \in C^{\infty}(\Omega)$; the reasoning is based on the Schauder fixed-point theorem.

Next, we specify a class of functions with certain regularity properties and use a compactness argument to establish the existence, in this class, of a solution to the original problem with piecewise constant initial density $\rho_{0}$. We then show the existence of a smooth surface separating the parts of the domain occupied by the two fluids.

Finally, we prove that the solution is unique.

\subsection{Smooth initial density: existence and uniqueness}

Throughout this subsection, we fix the initial density $\rho_{0} \in C^{\infty}(\Omega)$ and a number $q>n$.

The function class $\mathcal{M}$ consists of all $t$-continuous functions

$$
\widetilde{\rho} \in C\left(0, T ; L^{q}(\Omega)\right)
$$

such that the $L^{q}(\Omega)$-norm of $\widetilde{\rho}(\cdot, t)$ is bounded by the constant corresponding to the one-phase flow of the heavier fluid:

$$
\tilde{\rho} \in \mathcal{M} \Longleftrightarrow\left\{\begin{array}{l}
\left.\widetilde{\rho}\right|_{t=0}=\rho_{0}, \\
\max _{t \in(0, T)}\|\widetilde{\rho}(t)\|_{L^{q}(\Omega)} \leqslant \rho^{+}(\operatorname{mes} \Omega)^{1 / q} .
\end{array}\right.
$$

We fix the time range $T$ and define the following two linear operators. The first transforms a 'frozen' density into the corresponding field of velocities:

$$
\mathcal{M} \ni \widetilde{\rho} \mapsto U[\widetilde{\rho}] \in L^{\infty}\left(0, T ; W^{2, q}(\Omega)\right) .
$$

The other one describes the evolution of density driven by a 'frozen' velocity field (and starts from the initial smooth density specified in the beginning of the subsection):

$$
L^{\infty}\left(0, T ; W^{2, q}(\Omega)\right) \ni U \mapsto \underline{\rho}[U]=\rho_{\rho_{0}}[U] \in L^{q}\left(0, T ; L^{q}(\Omega)\right) .
$$

More precisely, the operator $U$ transforms $\widetilde{\rho}$ into the solution of

$$
\begin{aligned}
\triangle U & =\nabla p+\alpha \widetilde{\rho} \mathbf{e}, \\
\operatorname{div} U & =0,\left.\quad U\right|_{S}=0 .
\end{aligned}
$$

The operator $\rho=\rho_{\rho_{0}}$, which depends on the given initial density $\rho_{0}$, transforms $U$ into the solution of the Cauchy problem

$$
\frac{\partial \rho}{\partial t}+U \cdot \nabla \rho=0, \quad \rho(x, 0)=\rho_{0}(x) .
$$


For a smooth initial density $\rho_{0}$, the original problem of finding $u$ and $\rho$ from equations (1.1)-(1.3) reduces to finding a fixed point of the superposition of these two linear operators, the operator $F=\rho \circ U$ defined as

$$
\mathcal{M} \ni \widetilde{\rho} \mapsto F[\widetilde{\rho}]=\rho \circ U(\widetilde{\rho}) \stackrel{\text { def }}{=} \rho_{\rho_{0}}[U[\widetilde{\rho}]] \in \mathcal{M} .
$$

We will show that the conditions of the Schauder fixed-point theorem are satisfied for the 'quadratic' operator $F_{\rho_{0}}$.

2.1.1 Continuity of $F_{\rho_{0}}$. Below, we use notation $K_{i}$ for positive constants whose values depend on the shape of the domain $\Omega$ alone. We denote by $C, C_{i}$, etc., the positive constants dependent only on $T$, the larger density $\rho^{+}$and the shape of the domain $\Omega$, but not on the finer properties of $\widetilde{\rho}$. No attempt is made to keep track of their values, and same notation can be used to refer to different constants of these types in different parts of the arguments.

(a) For each function $\widetilde{\rho} \in \mathcal{M}$, the linear problem

$$
\begin{aligned}
\Delta u & =\nabla p+\alpha \tilde{\rho} e, \\
\operatorname{div} u & =0,\left.\quad u\right|_{S}=0,
\end{aligned}
$$

has* a unique solution $u \in L^{\infty}\left(0, T ; W^{2, q}(\Omega)\right)$.

For each $q \in(1, \infty)$ (and each value of the parameter $t \in[0, T]$ ), the solution admits the estimates (see $[4,10])$

$$
\|u(t)\|_{W^{2, q}(\Omega)} \leqslant K_{1}\|\widetilde{\rho}(t)\|_{L^{q}(\Omega)} \leqslant C_{1} .
$$

Combined with the embedding theorem for the pair of spaces $W^{2, q}(\Omega) \hookrightarrow C^{1, \lambda}(\bar{\Omega})$ (see [8] ), this estimate shows that for $q>n$ and $\lambda=1-\frac{n}{q}$

$$
\left\{\begin{array}{l}
u \in L^{\infty}\left(0, T ; C^{1, \lambda}(\bar{\Omega})\right), \\
\|u(t)\|_{C^{1, \lambda}(\Omega)} \leqslant K_{2}\|u(t)\|_{W^{2, q}(\Omega)} \leqslant C_{2} .
\end{array}\right.
$$

Whenever $\widetilde{\rho}$ is continuous with respect to $t$ as an $L^{q}(\Omega)$-valued function, the above estimates imply that $U$ is continuous as a function of $t$ with values in $W^{2, q}(\Omega)$ (or $C^{1, \lambda}(\Omega)$ ), or as a realvalued function of $t$ and $x$.

(b) We establish now the existence of a regular solution $\rho$ to the transport equation (2.3) for a smooth initial density $\rho_{0} \in C^{\infty}(\bar{\Omega})$ such that $\rho^{-} \leqslant \rho_{0}(x) \leqslant \rho^{+}$.

Given the velocity field $U$, we find the starting point $\hat{\xi}(t ; x) \in \Gamma(0)$ of the characteristic of (2.3) which hits $x$ at time $t$ :

$$
\begin{gathered}
\frac{\mathrm{d}}{\mathrm{d} t} X(t ; \xi)=U(X(t ; \xi), t), \quad X(0 ; \xi)=\xi, \\
X(t ; \widehat{\xi}(t, x))=x .
\end{gathered}
$$

For each $t>0$, the transformation $x \mapsto \widehat{\xi}$ is continuously differentiable in $x$ if $U$ enjoys the regularity typical of solutions to $(2.5)$ :

$$
\sup _{\Omega}\left|\nabla_{\xi} X(t ; \xi)\right|+\sup _{\Omega}\left|\nabla_{x} \widehat{\xi}(t, x)\right| \leqslant C_{3} .
$$

${ }^{*}$ These results originate in [14] and [7, 11, 12]; for details, see, e.g., [4]. 
To see this, it suffices to use the appropriate estimates for $\nabla U$ and note that the Jacobian $\left|\frac{\partial X}{\partial \xi}\right|$ preserves its value due to incompressibility.

The above provides for the existence of a unique solution of (2.3). Its explicit representation using (2.8) is [1]

$$
\left.\rho(x, t)=\rho_{0} \widehat{\xi}(x, t)\right) .
$$

It shows that $\rho(x, t)$ is uniformly bounded:

$$
0<\rho^{-} \leqslant \rho(x, t)=\rho_{0}(\widehat{\xi}(x, t)) \leqslant \rho^{+} .
$$

The density inherits from $\rho_{0}$ the existence of bounded derivatives in spatial variables

$$
|\nabla \rho(x, t)|=\left|\nabla \rho_{0} \|_{x=\widehat{\xi}(x, t)}\right| \nabla \widehat{\xi}(x, t)\left|\leqslant C_{3}\right| \nabla \rho_{0}(\widehat{\xi}(x, t)) \mid,
$$

where the constant could be expressed in terms of that in (2.7). Because of (2.3) and (2.7), this guarantees also the existence of a bounded time derivative:

$$
\sup _{(x, t)}\left(\left|\frac{\partial}{\partial t} \rho(x, t)\right|+|\nabla \rho(x, t)|\right) \leqslant C_{4}\left(\rho^{+}\right) \sup _{x}\left|\nabla \rho_{0}(x)\right| .
$$

(c) To see that the operator

$$
\widetilde{\rho} \mapsto U[\widetilde{\rho}] \mapsto F[\widetilde{\rho}]=\rho[U[\widetilde{\rho}]]
$$

of (2.4) is continuous, we consider the difference

$$
\bar{\rho}=\rho^{(1)}-\rho^{(2)}=F\left[\widetilde{\rho}^{(1)}\right]-F\left[\widetilde{\rho}^{(2)}\right]
$$

for two smooth densities $\widetilde{\rho}^{(i)}$ (note that the smooth initial density $\rho_{0}$ is the same in both cases). It solves the inhomogeneous transport equation

$$
\frac{\partial}{\partial t} \bar{\rho}=U^{(1)} \cdot \nabla \bar{\rho}-\nabla \rho^{(2)} \cdot\left(U^{(1)}-U^{(2)}\right), \quad U^{(i)}=U\left[\widetilde{\rho}^{(i)}\right]
$$

with the initial datum $\bar{\rho}(x, 0)=0$. It is obvious that

$$
\begin{aligned}
\sup _{(x, t)}|\bar{\rho}(x, t)| & \leqslant T \sup _{(x, t)}\left|\nabla \rho^{(2)}(x, t)\right| \\
& \times \sup _{(x, t)}\left|U^{(1)}(x, t)-U^{(2)}(x, t)\right| .
\end{aligned}
$$

The mapping $\widetilde{\rho} \mapsto F[\widetilde{\rho}]$ (cf. (2.4)) can also be considered as a bounded operator

$$
L^{q}\left(0, T ; L^{q}(\Omega)\right) \ni \widetilde{\rho} \mapsto F[\widetilde{\rho}] \in L^{q}\left(0, T ; L^{q}(\Omega)\right)
$$

in $L^{q}\left(0, T ; L^{q}(\Omega)\right)$. This operator is continuous because by estimates (2.6) and (2.7)

$$
\sup _{(x, t)}\left|U^{(1)}(x, t)-U^{(2)}(x, t)\right| \leqslant C_{5} \sup _{t \in[0, T]}\left\|\widetilde{\rho}^{(1)}(., t)-\widetilde{\rho}^{(2)}(., t)\right\|_{L^{q}(\Omega)},
$$

and consequently

$$
\begin{aligned}
\sup _{(x, t)}|\bar{\rho}(x, t)| & =\sup _{(x, t)}\left|F\left[\widetilde{\rho}^{(1)}\right](x, t)-F\left[\widetilde{\rho}^{(2)}\right](x, t)\right| \\
& \leqslant C_{6} \sup _{t \in[0, T]}\left\|\widetilde{\rho}^{(1)}(., t)-\widetilde{\rho}^{(2)}(., t)\right\|_{L^{q}(\Omega)} .
\end{aligned}
$$




\subsubsection{Compactness of $F_{\rho_{0}}$ and $W^{1,1}$ bounds for density.}

Bounds for density. $\quad$ By (2.11) and (2.12), the operator $F$ of (2.4) does indeed map $\mathcal{M}$ into itself continuously. The Schauder theorem requires compactness. This latter results in an obvious way from the smoothness of the initial density $\rho_{0}$ and (2.13), so the fixed point theorem is applicable.

However, it is worth while to do the calculations in more detail keeping in mind the subsequent passage to non-smooth initial densities that are merely functions of the bounded variation. During the process, one can use estimates in terms of $L^{1}$-norm of $\nabla \rho_{0}$ but not the $L^{\infty}$ bound of (2.13). For this reason, we need estimates for $\bar{\rho}$ (cf. (2.14)) in terms of the $B V$-norm of $\rho_{0}$. An estimate of $L^{q}$ norm of $\nabla \rho$ could be deduced from (2.12). Yet, it seems expedient to derive the desired inequality in a more straightforward manner.

For a smooth initial density $\rho_{0}$, both velocity and density are smooth, and the derivatives $\rho_{i}=$ $\nabla_{i} \rho$ satisfy the equations

$$
\frac{\partial \rho_{i}}{\partial t}+u \cdot \nabla \rho_{i}=-\sum_{j=1}^{n} \rho_{j} \nabla_{i} u_{j}, \quad \rho_{i}(x, 0)=\nabla_{i} \rho_{0}(x) .
$$

We multiply the $i$ th equation by $\phi_{i}=\rho_{i} / \sqrt{\rho_{i}^{2}+\delta^{2}}$ with $\delta>0$, and integrate over $\Omega_{T}=\Omega \times[0, T]$. The result is

$$
\begin{gathered}
\left.\int_{\Omega} \sqrt{\rho_{i}^{2}+\delta^{2}} \mathrm{~d} x\right|_{0} ^{t}+\int_{0}^{t} \int_{\Omega} u \cdot \nabla \Psi\left(\rho_{i}\right) \mathrm{d} x \mathrm{~d} t \\
=-\int_{0}^{t} \int_{\Omega} \sum_{j=1}^{n} \nabla_{i} u_{j} \frac{\rho_{i} \rho_{j}}{\sqrt{\rho_{i}^{2}+\delta^{2}}} \mathrm{~d} x \mathrm{~d} t,
\end{gathered}
$$

where $\Psi\left(\rho_{i}\right)=\int_{0}^{\rho_{i}}\left(s^{2}+\delta^{2}\right)^{-1 / 2} \mathrm{~d} s$. Since $u$ is solenoidal and vanishes on boundary,

$$
\begin{aligned}
\int_{\Omega} u \cdot \nabla \Psi\left(\rho_{i}\right) \mathrm{d} x= & -\int_{\Omega} \Psi\left(\rho_{i}\right) \mathrm{div} u \mathrm{~d} x \\
& +\int_{S} \Psi\left(\rho_{i}\right) u \cdot \mathbf{n} \mathrm{d} S=0
\end{aligned}
$$

(here $\mathbf{n}$ is the unit normal to $S$ ). Now, we pass to the limit as $\delta \searrow 0$ and get the equalities

$$
\begin{aligned}
\left.\int_{\Omega}\left|\rho_{i}\right| \mathrm{d} x\right|_{0} ^{t} & =\left.\lim _{\delta \searrow 0} \int_{\Omega} \sqrt{\rho_{i}^{2}+\delta^{2}} \mathrm{~d} x\right|_{0} ^{t} \\
& =\lim _{\delta \searrow 0}\left(-\int_{0}^{t} \int_{\Omega} \sum_{j=1}^{n} \nabla_{i} u_{j} \frac{\rho_{i} \rho_{j}}{\sqrt{\rho_{i}^{2}+\delta^{2}}} \mathrm{~d} x \mathrm{~d} t\right) \\
& =-\int_{0}^{t} \int_{\Omega} \sum_{j=1}^{n} \operatorname{sign}\left(\rho_{i}\right) \rho_{j} \nabla_{i} u_{j} \mathrm{~d} x \mathrm{~d} t .
\end{aligned}
$$

One can sum these identities in $i$ and differentiate in $t$ to arrive at the equation

$$
\frac{\mathrm{d}}{\mathrm{d} t} \int_{\Omega} \sum_{i=1}^{n}\left|\rho_{i}(x, t)\right| \mathrm{d} x=-\int_{\Omega} \sum_{i, j=1}^{n} \rho_{j} \nabla_{i} u_{j} \operatorname{sign}\left(\rho_{i}\right) \mathrm{d} x .
$$


It follows that

$$
\frac{\mathrm{d}}{\mathrm{d} t} \int_{\Omega} \sum_{j=1}^{n}\left|\rho_{j}(x, t)\right| \mathrm{d} x \leqslant \max _{(x, t)}|\nabla u(x, t)| \int_{\Omega} \sum_{j=1}^{n}\left|\rho_{j}(x, t)\right| \mathrm{d} x .
$$

Applying the Gronwall inequality, we establish that for each $t \in[0, T]$

$$
\|\nabla \rho(t)\|_{L^{1}(\Omega)} \leqslant C_{7}\left\|\nabla \rho_{0}\right\|_{L^{1}(\Omega)} .
$$

By the transport equation (1.3)

$$
\left\|\frac{\partial}{\partial t} \rho(t)\right\|_{L^{1}(\Omega)} \leqslant \max _{(x, t)}|u(x, t)|\left\|\nabla \rho_{0}\right\|_{L^{1}(\Omega)} .
$$

Combining this estimate and (2.13), we conclude finally that

$$
\begin{gathered}
\max _{t}\|\rho(t)\|_{L^{\infty}(\Omega)} \leqslant \rho^{+}, \\
\max _{t}\left(\left\|\frac{\partial \rho}{\partial t}(t)\right\|_{L^{1}(\Omega)}+\|\nabla \rho(t)\|_{L^{1}(\Omega)}\right) \leqslant C_{8}\left\|\nabla \rho_{0}\right\|_{L^{1}(\Omega)} .
\end{gathered}
$$

By (2.17), $F$ is continuous. Estimates (2.21) and (2.22) guarantee that the operator $F: \mathcal{M} \rightarrow$ $\mathcal{M}$ is compact and, therefore, has at least one fixed point, which defines the solution of the original problem (1.1)- (1.5) for a smooth initial density $\rho_{0}(x)$.

Regularity of velocity as function of time. In the later analysis, it will be important that the velocity field enjoys a certain degree of smoothness with respect to the 'time parameter' $t$. One can deduce from (2.7), (2.3), and (2.21), (2.22) that velocity is Hölder continuous as a $W^{2, q}(\Omega)$-valued function of $t$. To prove this, consider the equations that relate the difference

$$
\delta u=u(x, t+\delta t)-u(x, t)
$$

to the corresponding increment of the density $\delta \rho=\rho(x, t+\delta t)-\rho(x, t)$.

It is easily seen that the function $\delta u$ solves the stationary Stokes system (2.5) with $\widetilde{\rho}=\delta \rho$. As before, whatever the choice of $q \in(1, \infty)$, it admits estimates similar to (2.7), namely,

$$
\|\delta u(t)\|_{C^{1, \lambda}(\Omega)} \leqslant K_{2}\|\delta \rho(t)\|_{L^{q}(\Omega)} .
$$

Using (2.21)-(2.22) and (2.23), we see that the velocity, considered as a $C^{1+\lambda}(\Omega)$-valued function of time, is Hölder continuous:

$$
\|u(t+\delta t)-u(t)\|_{C^{1+\lambda}(\Omega)} \leqslant K_{2}\left(2 \rho^{+}\right)^{\frac{q-1}{q}}\|\delta \rho(t)\|_{L^{1}(\Omega)}^{\frac{1}{q}} \leqslant C_{9}|\delta t|^{\frac{1}{q}}
$$

\subsection{Passage to non-smooth initial data}

In the case of piecewise constant initial density, let us approximate the function $\rho_{0}(x)$ by smooth functions $\rho_{0}^{(m)} \in C^{\infty}(\bar{\Omega})$ such that $\rho_{0}^{(m)}=\rho_{0}$ outside a $\frac{1}{m}$-neighbourhood of $\Gamma_{0}$ and

$$
\left\|\rho_{0}-\rho_{0}^{(m)}\right\|_{L^{q}(\Omega)} \rightarrow 0, \quad m \rightarrow \infty, \quad\left\|\rho_{0}^{m}\right\|_{W^{1,1}(\Omega)} \leqslant M_{2},
$$


which is possible by the assumption that the initial free boundary $\Gamma_{0}$ is a surface of class $C^{2}$.

Let $u^{(m)}, \rho^{(m)}$ be the solution of problem (1.1)-(1.5) that corresponds to the initial density $\rho_{0}^{(m)}$. The estimates (2.21), (2.22) allow us to extract a subsequence $\left\{\rho^{(m)}\right\}$ which converges strongly in $L_{r}\left(\Omega_{T}\right)$ for some $r>1$. This can be done by the embedding theorem for the pair of spaces $W^{1,1}\left(\Omega_{T}\right) \hookrightarrow L_{r}\left(\Omega_{T}\right)$.

Since the densities $\rho^{(m)}(x, t)$ satisfy (2.21), (2.22), the same subsequence $\left\{\rho^{(m)}\right\}$ converges strongly in $L^{\infty}\left(0, T ; L^{q}(\Omega)\right)$ for each $q>n$. According to estimates (2.6), this implies the strong convergence of the corresponding subsequence of velocities $\left\{u^{(m)}\right\}$ in the space $L^{\infty}\left(0, T ; W^{2, q}(\Omega)\right)$. Estimates (2.7)) guarantee strong convergence of $\left\{u^{(m)}\right\}$ in the space $L^{\infty}\left(0, T ; C^{1, \lambda}(\bar{\Omega})\right)$ with $\lambda=1-\frac{n}{q}$ as well.

Passing to the limit in the corresponding equations (1.1)-(1.3) for the functions $u^{(m)}, \rho^{(m)}$, and $p^{(m)}$ as $m \rightarrow \infty$, one sees that the limit functions satisfy the original problem. Instead of the original transport equation (1.3), the density $\rho$ satisfies the corresponding integral identity

$$
\begin{aligned}
\int_{\Omega_{T}} \frac{\mathrm{d} \varphi}{\mathrm{d} t} \rho \mathrm{d} x \mathrm{~d} t & \equiv \int_{\Omega_{T}}\left(\frac{\partial \varphi}{\partial t}+u \cdot \nabla \varphi\right) \rho \mathrm{d} x \mathrm{~d} t \\
& =-\int_{\Omega} \rho_{0}(x) \varphi(x, 0) \mathrm{d} x
\end{aligned}
$$

for any smooth test function $\varphi$ such that $\varphi(x, T)=0$ and $\left.\varphi\right|_{S}=0$.

\subsection{Existence of a regular free boundary}

Let us prove now that there exists a regular free boundary which divides the domain $\Omega$ into two subdomains $\Omega^{+}(t)$ and $\Omega^{-}(t)$ such that

$$
\rho(x, t)= \begin{cases}\rho^{+}, & x \in \Omega^{+}(t), \\ \rho^{-}, & x \in \Omega^{-}(t) .\end{cases}
$$

The reasoning is based on the explicit representation of the solution to the transport equation (1.3) using translation along its characteristics

$$
\frac{\mathrm{d} X}{\mathrm{~d} t}=u(X(\xi, t), t), \quad X(\xi, 0)=\xi, \quad \xi \in \Gamma_{0},
$$

with the velocity field $u$ from the solution of the original problem (cf. [10]).

Note that the characteristics $X^{(m)}(\xi, t)$ of the transport equation (1.3) for the smooth density $\rho^{(m)}$, which corresponds to $\rho_{0}^{(m)}$ of (2.25), converge uniformly to those defined by (2.27). For this reason, whenever a point $x$ is not in $\Gamma(t)=\left\{X(\xi, t): \xi \in \Gamma_{0}\right\}$, it has a neighbourhood

$$
U_{\delta}(x) \subseteq\left\{X(\xi, t): \rho_{0}(\xi)=\text { const }, \quad \operatorname{dist}\left(\xi, G_{0}\right) \geqslant \delta\right\}, \quad \delta>0
$$

separated from $\Gamma(t)$ by a positive distance. For $m$ large enough, each characteristic $X^{(m)}$ that arrives at $U_{\delta}(x)$ at time $t$ starts from a point $\xi$ with the same initial value of density $\rho_{0}^{(m)}(\xi)=\rho_{0}(\xi)$, so $\rho^{(m)}$ is constant on $U(x)$. By strong $L^{q}$-convergence of densities, the limit $\rho(x, t)$ is also constant

\footnotetext{
We preserve the same notation for this subsequence for the sake of simplicity.
} 
on $U_{\delta}(x)$ and assumes one of the values $\rho^{ \pm}$. Thus, the complement in $\Omega$ to $\Gamma(t) \cup S$ consists of two open sets $\Omega^{ \pm}(t)=\left\{\rho(x, t)=\rho^{ \pm}\right\}$.

To show that the moving boundary $\Gamma(t)$ is a smooth surface, we consider in more detail characteristics (2.27) that start from the initial surface $\Gamma(0)=\Gamma_{0}$. By estimate (2.7) the velocity field is $C^{1, \lambda}$ smooth, and it remains to show that the corresponding surface $\Gamma(t)=\{x: x=X(\xi, t)\}$ belongs to the same class $C^{1, \lambda}$.

Differentiating (2.27) in parameter $\xi$, we get a Cauchy problem for the derivatives $\left(\partial / \partial \xi_{j}\right) X_{k}$ :

$$
\frac{\mathrm{d}}{\mathrm{d} t}\left(\frac{\partial X_{i}}{\partial \xi_{j}}\right)-\sum_{k=1}^{n} \nabla_{k} u_{i} \frac{\partial X_{k}}{\partial \xi_{j}}=0,\left.\quad \frac{\partial X_{i}}{\partial \xi_{j}}\right|_{t=0}=\delta_{i j},
$$

where $\delta_{k}^{j}$ is the Kronecker symbol. This is one more transport equation, so the derivatives considered are bounded for $t>0$.

We arrive at a similar conclusion considering the quotients of finite differences

$$
M_{i j}^{s}=\frac{1}{h^{\lambda}}\left(\frac{\partial X_{i}}{\partial \xi_{j}}\left(t ; \xi+h \mathbf{e}_{s}\right)-\frac{\partial X_{i}}{\partial \xi_{j}}(t ; \xi)\right),
$$

where $\left(\mathbf{e}_{1}, \ldots, \mathbf{e}_{n}\right)$ is the standard basis in $\mathbb{R}^{n}$. The pertinent Cauchy problem is

$$
\frac{\mathrm{d}}{\mathrm{d} t} M_{i j}^{s}=\sum_{k=1}^{n} A_{i k}^{s} M_{k j}^{s}+B_{i j}^{s}, \quad M_{i j s}(\xi, 0)=0,
$$

and the coefficients

$$
\begin{aligned}
A_{i k}^{s} & =\nabla_{k} u_{i}\left(X\left(t, \xi+h \mathbf{e}_{s}\right),\right. \\
B_{i j}^{s} & =\frac{1}{h^{\lambda}} \sum_{k=1}^{n}\left(\nabla_{k} u_{i}\left(X\left(t ; \xi+h \mathbf{e}_{s}\right), t\right)-\nabla_{k} u_{i}(X(t ; \xi), t)\right) \frac{\partial X_{k}}{\partial \xi_{j}}(t ; \xi) .
\end{aligned}
$$

are bounded. Therefore $\left|M_{k j}^{S}\right|$ are bounded, and $\partial X_{k} / \partial \xi_{j}$ are Hölder continuous in parameter $\xi$. Consequently, the map induced by (2.27) is smooth for each $t>0$ :

$$
x=X(\xi, t) \in C^{1+\lambda} .
$$

Note that for incompressible fluids the Jacobian $J(t)=\operatorname{det}\left(\frac{\partial X}{\partial \xi}\right)$ of the map (2.27) preserves its value:

$$
J(t)=J(0)=1, \quad t \in[0, T],
$$

by (1.2) and the Euler formula

$$
\frac{\mathrm{d}}{\mathrm{d} t} J(t)=\rho \operatorname{div} u .
$$

Thus, we can use (2.28) to obtain for all $t \in[0, T]$ and $\left(\xi_{1}, \xi_{2}\right) \in \Omega_{0}$ the estimates

$$
\frac{1}{C}\left|\xi_{1}-\xi_{2}\right| \leqslant\left|X\left(\xi_{1}, t\right)-X\left(\xi_{2}, t\right)\right| \leqslant C\left|\xi_{1}-\xi_{2}\right|
$$

with a positive constant $C$. This means that at any time $t>0$ the free boundary has no common points with the given boundary if the two boundaries had no common points at the initial moment $t=0$. 


\subsection{Uniqueness of the solution}

The last step in the proof of Theorem 1.1 is to show that the solution of the boundary-and-initialvalue problem (1.1)-(1.5) is unique.

Suppose that the problem has two solutions $\left(u^{(i)}, p^{(i)}, \rho^{(i)}\right), i=1,2$. Let us consider the boundary-value problem for their difference

$$
u=u^{(1)}-u^{(2)}, \quad p=p^{(1)}-p^{(2)}, \quad \rho=\rho^{(1)}-\rho^{(2)} .
$$

The difference satisfies the equations

$$
\begin{aligned}
\Delta u & =\nabla p+\alpha \rho \mathbf{e}, \\
\operatorname{div} u & =0,\left.\quad u\right|_{S}=0, \\
\frac{\partial \rho}{\partial t}+u^{(1)} \cdot \nabla \rho & =-\operatorname{div}\left(\rho^{(2)} u\right), \quad \rho(x, 0)=0,
\end{aligned}
$$

where the last equation is fulfilled as an integral identity: for any smooth function $\varphi$ such that $\varphi(x, T)=0$

$$
I_{0} \equiv-\int_{0}^{T} \int_{\Omega} \rho \frac{\mathrm{d} \varphi}{\mathrm{d} t} \mathrm{~d} x \mathrm{~d} t=\int_{0}^{T} \int_{\Omega} \rho^{(2)}(u \cdot \nabla \varphi) \mathrm{d} x \mathrm{~d} t \equiv I_{1},
$$

and the full derivative $(\mathrm{d} / \mathrm{d} t) \varphi=(\partial / \partial t) \varphi+u^{(1)} \cdot \nabla \varphi$ is calculated along the velocity field $u^{(1)}$.

Using the fact that $\rho^{(2)}$ is piecewise constant, one can rewrite the term $I_{1}$ as

$$
\begin{aligned}
I_{1} & =\int_{\rho^{(2)}=\rho^{+}} \rho^{+}(u \cdot \nabla \varphi) \mathrm{d} x \mathrm{~d} t+\int_{\rho^{(2)}=\rho^{-}} \rho^{-}(u \cdot \nabla \varphi) \mathrm{d} x \mathrm{~d} t \\
& =\beta \int_{0}^{T} \mathrm{~d} t \int_{\Gamma^{(2)}(t)} \varphi(u \cdot \mathbf{n}) \mathrm{d} s,
\end{aligned}
$$

where $\beta=\rho^{+}-\rho^{-}$and $\mathbf{n}$ is the normal unit vector to the surface $\Gamma^{(2)}(t)$ which divides the domain $\Omega$ into two subdomains $\Omega^{+}(t)$ and $\Omega^{-}(t), \rho^{(2)}(x, t)=\rho^{ \pm}$in $\Omega^{ \pm}(t)$.

On the other hand, one can use the Lagrangian coordinates $(\xi, t) \rightarrow(x, t)$ defined by characteristics of the transport equation in (2.29):

$$
\frac{\mathrm{d} x}{\mathrm{~d} t}=u^{(1)}(x, t), \quad x(\xi, 0)=\xi .
$$

In Lagrangian coordinates, the integral $I_{0}$ becomes

$$
I_{0}=-\int_{0}^{T} \int_{\Omega} \widehat{\rho}(\xi, t) \frac{\partial \widehat{\varphi}}{\partial t}(\xi, t) \mathrm{d} \xi \mathrm{d} t
$$

(here $\widehat{\rho}(\xi, t)=\rho(x(\xi, t), t)$ and $\widehat{\varphi}(\xi, t)=\varphi(x(\xi, t), t)$ ).

As in the proof of Lemma 4.1 in [8: Chapter 3], we choose the 'Lagrangian' test function as the time average

$$
\widehat{\varphi}(\xi, t)=\widehat{\eta}_{\bar{h}}(\xi, t)=\frac{1}{h} \int_{t-h}^{t} \widehat{\eta}(\xi, \tau) \mathrm{d} \tau
$$


and put

$$
\widehat{\rho}_{h}(\xi, t)=\frac{1}{h} \int_{t}^{t+h} \widehat{\rho}(\xi, \tau) \mathrm{d} \tau .
$$

It is easily seen that

$$
I_{0}=-\int_{\Omega_{T}} \widehat{\rho}_{h}(\xi, t) \frac{\partial \widehat{\eta}}{\partial t}(\xi, t) \mathrm{d} \xi \mathrm{d} t=\int_{\Omega_{T}} \widehat{\eta}(\xi, t) \frac{\partial \widehat{\rho}_{h}}{\partial t}(\xi, t) \mathrm{d} \xi \mathrm{d} t .
$$

Arguing along the customary lines, we can choose for the test function $\widehat{\eta}$ in (2.30) any bounded function $\widehat{\psi}$ which vanishes for $t>t_{0}, t_{0}<T-h$. For $\widehat{\psi}=\operatorname{sign} \widehat{\rho}_{h}(\xi, t)$, identity (2.30) becomes

$$
I_{0}=\int_{\Omega}\left|\widehat{\rho}_{h}\left(\xi, t_{0}\right)\right| \mathrm{d} \xi=\int_{\Omega}\left|\rho_{h}\left(x, t_{0}\right)\right| \mathrm{d} x .
$$

Therefore

$$
\int_{\Omega}\left|\rho_{h}\left(x, t_{0}\right)\right| \mathrm{d} x \leqslant I_{1} \leqslant \alpha\left|\int_{\Gamma_{t_{0}}^{(2)}} \varphi(u \cdot \mathbf{n}) \mathrm{d} s \mathrm{~d} t\right| \leqslant K_{1} \int_{0}^{t_{0}}\|u(t)\|_{W^{1,1}(\Omega)} \mathrm{d} t .
$$

Next, we pass to the limit as $h \rightarrow 0$. We finally get the inequality

$$
\left\|\rho\left(t_{0}\right)\right\|_{L^{1}(\Omega)}=\int_{\Omega}\left|\rho\left(x, t_{0}\right)\right| \mathrm{d} x \leqslant K_{1} \int_{0}^{t_{0}}\|u(t)\|_{W^{1,1}(\Omega)} \mathrm{d} t .
$$

We consider once again the Stokes equations for the function $u$. One can see that the force term $g=\alpha \rho \mathbf{e}$ defines a continuous linear functional in the space $W^{1, r}(\Omega)$ for $r>n$. Indeed,

$$
\begin{aligned}
|\langle g \cdot u\rangle| & =\left|\int_{\Omega} g \cdot u \mathrm{~d} x\right|=\left|\alpha \int_{\Omega} \rho(\mathbf{e} \cdot u) \mathrm{d} x\right| \\
& \leqslant K_{2}\|\rho(t)\|_{L^{1}(\Omega)} \max _{x \in \Omega}|u(x, t)| \leqslant K_{3}\|\rho(t)\|_{L^{1}(\Omega)}\|u(t)\|_{W^{1, r}(\Omega)} .
\end{aligned}
$$

Therefore (see [4: p.226]) $g \in W_{0}^{-1, r^{*}}(\Omega)$, and consequently $u(t) \in W^{1, r^{*}}(\Omega)$, where $1<r^{*}=$ $\frac{r}{r-1}<n$. Moreover, $u(t)$ satisfies the inequality

$$
\|u(t)\|_{W^{1, r^{*}}(\Omega)} \leqslant K_{4}\|\rho(t)\|_{L^{1}(\Omega)} .
$$

Combined with (2.31), this estimate yields the inequality

$$
\frac{\mathrm{d}}{\mathrm{d} t}\|\rho(t)\|_{L^{1}(\Omega)} \leqslant K_{5}\|\rho(t)\|_{L^{1}(\Omega)},
$$

which shows that $\rho=0$.

Thus, the solution of the original problem (1.1)-(1.5) is unique.

\section{Acknowledgements}

This work is supported by Project PRAXIS XXI/2/2.1/MAT/53/94 'Modelos Matemáticos de Sistemas com Fronteiras Livres'. 


\section{REFERENCES}

1. Antontsev, S. N., Kazhikhov, A. V., \& Monakhov, V. N. Boundary Value Problems in Mechanics of Nonhomogeneous Fluids. North-Holland, Amsterdam (1990) pp. 309.

2. BöHM, M. On a nonhomogeneous Bingham fluid. J. Diff. Eq. 60, (1985) 259-284.

3. Cattabriga, L. Su un problema al contorno relativo al sistema di equazioni di Stokes. Rend. Sem. Mat. Padova 31, (1961) 308-340.

4. Galdi, G. P. An Introduction to the Mathematical Theory of the Navier-Stokes Equations, vol. 1. Springer-Verlag, New York (1994).

5. Giga, Y. \& TAKAHASHI, S. On global weak solutions of the nonstationary two-phase Stokes flow. SIAM J. Math. Anal. 25, (1994) 876-893.

6. Fernández-CARA, E., Guillén, F., \& Ortega, R. R. Some theoretical results for visco-plastic and dilatant fluids with variable density. Nonlinear Methods and Appl. 28, (1997) 1079-1100.

7. Ladyzhenskaya, O. A. Mathematical Problems in Viscous Incompressible Fluid Dynamics. Nauka, Moscow (1970). (English transl. of 1st edn. The Mathematical Theory of Viscous Incompressible Flow, 2nd edn. Gordon and Breach, New York (1969)).

8. Ladyzhenskaya, O. A., Solonnikov, V. A., \& Ural'Ceva, N. N. Linear and Quasi-Linear Parabolic Equations. Nauka, Moscow (1967). (English transl. Linear and Quasilinear Equations of Parabolic Type, Translations of Mathematical Monographs, vol. 23. American Mathematical Society, Providence, Rhode Island (1988)).

9. Nouri, A. \& Poupaud, F. An existence theorem for the multifluid Navier-Stokes problem. J. Diff. Eq. 122, (1995) 71-88.

10. Solonnikov, V. A. \& Ladyzhenskaya, O. A. On unique solvability of an initial-boundary value problem for viscous nonhomogeneous fluids. Zapiski Nauchn. Semin. LOMI 52, (1975) 52-109. (English transl.. J. Soviet Math. 9, (1978) 697-749.)

11. Maremonti, P. \& Solonnikov, V. A. Estimates for the Laplace operator in exterior domains. Zap. Nauchn. Sem. LOMI 146, (1985) 92-102.

12. Maremonti, P. \& Solonnikov, V. A. Su uma diseguaglianza per le soluzioni del problema di Stokes in domini esterni. Preprint. Dipart. di Matemat. Univ. di Napoli, (1986).

13. SIMON, J. Nonhomogeneous viscous incompressible fluids: existence of velocity, density, and pressure. SIAM J. Math. Anal. 21, (1990) 1093-1117.

14. Solonnikov, V. A. Estimates for solutions of nonstationary Navier-Stokes equations. Zap. Nauchn. Sem. LOMI 38, (1973) 153-231. (English transl. in J. Soviet Math. 8, (1977) 467-528.)

15. Yıн, C.-S. Dynamics of Nonhomogeneous Fluids. Collier-Macmillan Ltd., London (1965). 